\title{
A Theoretical Approach to The Implementation of Low-Voltage Smart Switch Boards
}

\author{
Péter Holcsik $^{1}$, Judith Pálfi ${ }^{1}$, Zsolt Čonka ${ }^{2}$, Mihai Avornicului ${ }^{3}$ \\ ${ }^{1}$ Óbuda University, Kandó Kálmán Faculty of Electrical Engineering, \\ Research Group of Applied Disciplines and Technologies in Energetics, \\ 1034 Budapest, Bécsi út 96, Hungary, peter.holcsik@elmu.hu, \\ palfi.judith@kvk.uni-obuda.hu \\ ${ }^{2}$ Technical University of Košice, Department of Electric Power Engineering, \\ Faculty of Electrical Engineering and Informatics, 04001 Košice, \\ Slovak Republic, zsolt.conka@tuke.sk \\ ${ }^{3}$ Babeş-Bolyai University, Cluj-Napoca, Faculty of Economics and Business \\ Administration, TeodorMihali street, Nr. 58-60. Campus UBBFSEGA 400591, \\ Cluj-Napoca, Romania, mihai.avornicului@econ.ubbcluj.ro
}

\begin{abstract}
Technological advances have made possible the fault location detection on the low-voltage distribution network using the fault location determination algorithm (FLDa). The results obtained by operating this algorithm can be implemented into a system that schedules the faults toward the electrician teams in charge of the troubleshooting. This solution, however, only addresses the processing and evaluation of signals based on remote signaling and does not provide the possibility of automatic interventions. This present paper investigates and describes the possibilities of automatic interventions on low-voltage distribution networks. This paper examines the Smart Switchboard concept developed by the Research Group of Applied Disciplines and Technologies in Energetics.
\end{abstract}

Keywords: theory; low-voltage distribution network; smart switchboard

\section{Introduction}

The basic task of electricity supply is to ensure safe and continuous service of the electrical networks. "The joint fulfillment of the requirements of safety, quality, and economic efficiency is a task based on compromises that represent the central issue of system management" [1]. In parallel with increasing consumer demands, power suppliers have to maintain the quality of their services on an adequate level. If not, regulatory sanctions would be applicable. 
"Electricity suppliers use several indicators for measuring the quality of electricity networks. The Hungarian Energy and Public Utility Regulatory Authority are following two indicators and expects their improvement by Hungarian electricity suppliers. These two indicators are the System Average Interruption Duration Index $(S A I D I)$ and the System Average Interruption Frequency Index (SAIFI)" [2, 3].

The System Average Interruption Frequency Index (SAIFI) shows the number of unscheduled outages for a consumption site in a specific interval (usually yearly), i.e., "the frequency of unplanned supply interruption per consumer" [4].

The SAIDI network quality indicator is given by:

$S A I D I=\frac{\sum_{i=1}^{n}\left(U_{i} \cdot N_{i}\right)}{N_{T}}[\mathrm{sec}]$

where $N_{i}$ is the number of customers and $U_{i}$ is the annual outage time for location $i$, and $N_{T}$ is the total number of customers served.

In other words,

$S A I D I=\frac{\text { sum of all customer interruption durations }}{\text { total number of customers served }}$

The System Average Interruption Duration Index (SAIDI) shows the average number of outage minutes per consumer, i.e., "the average duration of unplanned supply interruptions" [4].

The SAIFI network quality indicator is given by:

$S A I F I=\frac{\sum_{i=1}^{n}\left(\lambda_{i} \cdot N_{i}\right)}{N_{T}}[\sec ]$

where $\lambda_{i}$ is the failure rate, $N_{i}$ is the number of customers for location $i$ and $N_{T}$ is the total number of customers served. In other words,

SAIFI $=\frac{\text { total number of customer interruptions }}{\text { total number of customers served }}$

One of the SAIDI, SAIFI determinants of the network quality indicators is the number of consumers $\left(N_{i}\right)$ affected by the malfunction $i$. Energy supplier companies keep records of the number of consumers affected by the failure of a particular equipment. Due to these records, they are able to produce accurate accounts about the number of consumers left without service due to a specific network component failure [5].

Quality indicators are usually calculated per year. Thus, the number of customers $\left(N_{T}\right)$ used in the calculation of indicators is a constant value (number) determined for a specific year and a specific power supplier at the beginning of the year. This customary method is necessary in order to eliminate the effects of ongoing changes during the year and for carrying out uniform and transparent calculations [6]. 
The calculation method of the SAIDI index reflects that one of the most significant factors of the network quality indicator consists in the time interval of the failure, $i$ which starts from its detection (security operation or the first consumer report) and ending with the restoration of the service at the consumption point (this does not necessarily mean the restoration of the normal functioning) [7].

Hungarian legislation on electricity providers defines short-term network failures for which the time of interruption and the number of affected consumers are not included in the calculation of the SAIDI and SAIFI. The short-term network failures are the consumer interruptions of a maximum duration of 3 minutes in the following situations:

1. normal operational interruptions not exceeding the restart duration of the network automatics;

2. inefficient restarts in rigidly earthed networks not leading to consumer interruptions;

3. efficient functioning of non-UPS switch automatics and of current transformer switchback automatics. [8]

The SAIDI and SAIFI network quality indices can be influenced differently by the operational management levels of the medium (MV) and high-voltage (HV) distribution networks. These effects will be detailed in the following chapters. In the paper after presenting the possibilities of the MV and HV the authors will then present the new possibilities that are available on the LV network. The authors demonstrate the theoretical demonstration of the effectiveness of the solution developed by the Research Group of Applied Disciplines and Technologies in Energetics [2, 3, 5, 8, 9, 14].

\section{Breakdown Recovery of High-Voltage Transmission Networks}

"European power systems are constructed hierarchically and can be divided into three distinct parts: 750, 400, 220, and $120 \mathrm{kV}$ high-voltage transmission networks (HV), 35, 20, and $11 \mathrm{kV}$ medium-voltage distribution networks (MV) and 0,4 kV low-voltage distribution networks (LV)" $[9,18]$. The construction and operation of the HV, MV, and LV networks is significantly different from each other influencing the troubleshooting method.

High-voltage transmission networks are looped. Their theoretical operation schematics is represented in Figure 1 [10]. 


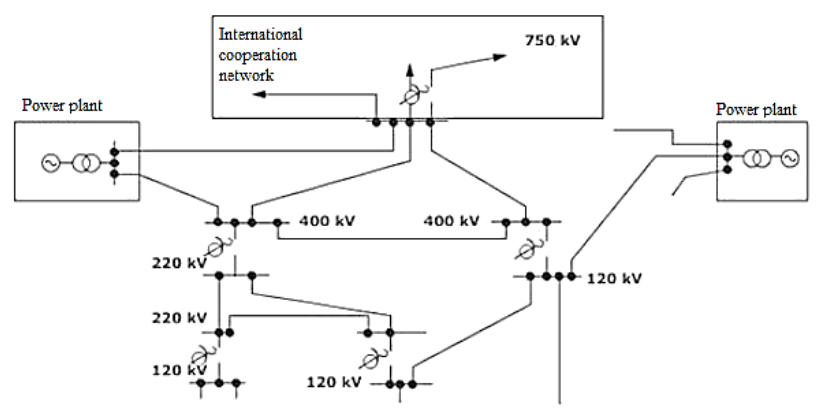

Figure 1

Theoretical schematics of a looped network [10]

The basic feature of the looped network is that there are various connections operating simultaneously in different directions between the various feeding and consumer points (Figure 1). The consumers joined to the looped network can be fed from many sides and through various routes. Hence, the looped network operates with maximum reliability. Another advantage is that multiple power routes (connectivity statuses) can be realized granting optimal power supply to the individual consumers (optimal operational parameters, minimum loss and low voltage drop) [10].

\subsection{The $n$-1Principle}

The looped design of the high voltage transmission network enables the realization of the $n-1$ criteria. According to the $n-1$ principle, the transmission system is constructed in such a way that the malfunction of element 1 of the system does not cause any loss at the consumer level (Figure 2).

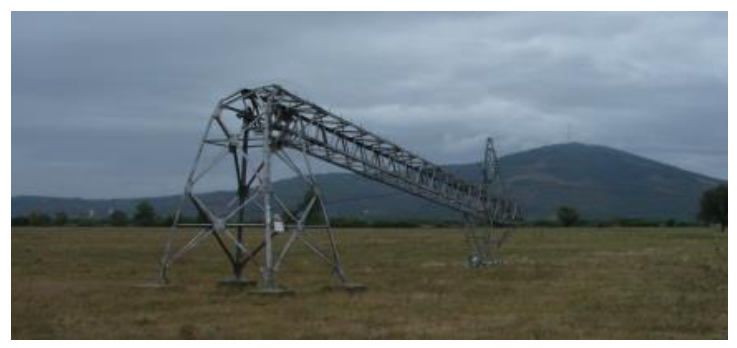

Figure 2

The identified fault location of the HV operational failure not resulting in an outage [11]

Figure 2 shows the malfunction of a high-voltage network which did not result in a failure for the consumers. In areas where enhanced safety is required (for example in the vicinity of a nuclear power station) the compliance with the $n-2$ criteria must be ensured [1]. 


\subsection{Fault Recovery IT Support for the High-Voltage Transmission Network}

The breakdown of high-voltage transmission networks can affect up to 100,000 individual consumers. Hence, along with the structural design, many other forms of assistance collectively named as IT support have been implemented in the operation of the HV networks.

IT support requires the constant transfer, storage and processing of large- and mostly real-time data. Online functions supporting the operational system control can be divided into two groups according to their complexity and use. These are the SCADA (Supervisory Control and Data Acquisition System) and the EMS (Energy Management System) functions.

Below are listed some of the typical functions of the SCADA system.

1. Reception of remote measurements and signals, e.g., real and reactive performance flows, busbar voltages, frequency measurements, breaker and disconnector position indicators, gear position of transformer regulators, etc.

2. Real time database creation with short refresh times, of usually a couple of seconds.

3. Representation, man-machine relation: the cyclically refreshed information usually appears on screens and on schema tables.

4. Registering and archiving.

5. Observing the limit values and gradients, recognizing endangered and dangerous states.

6. Topology analysis, inspection of the connection status and of the network continuity, registering changes, recognizing failures.

7. Issuing remote commands. The commands of the controlling personnel and the value settings calculated by the EMS and approved for dispatch are transmitted through the SCADA tele-mechanics system to the controlled objects [12].

Some of the typical EMS functions are:

1. automatic generation control (AGC),

2. load-flow or power flow,

3. real time sequence,

4. Model Update (MU),

5. State Estimation (SE)

6. Voltage Scheduler (VS), Automatic Voltage Control (AVC).

7. Operator Training Simulation (OTS). [12] 


\section{Breakdown Recovery of Medium-Voltage Distribution Networks}

The operation of the medium-voltage distribution network is radial. However, the topology of their design is partially looped. Therefore, on the $10 \mathrm{kV}$ urban cable network and on the so-called main line sections of the $20 \mathrm{kV}$ and $35 \mathrm{kV}$ overhead line networks, the electricity supply to consumers can be temporarily ensured through transfers without the correction of failures [9, 13]. This partially looped solution can be dubbed as a ringed or a curbed network, according to its design:

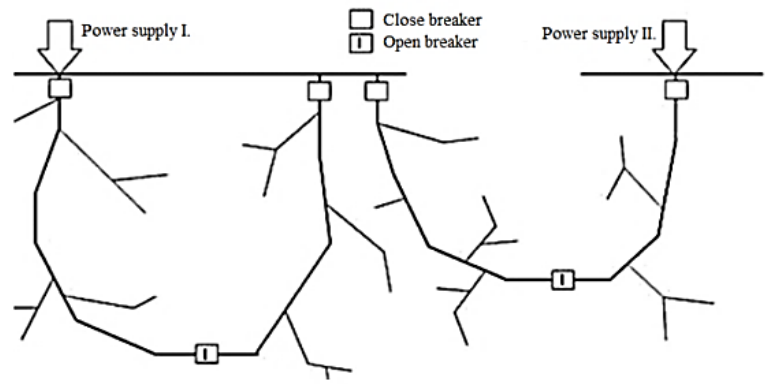

Figure 3

Ringed and curved networks [11]

Figure 3 shows a ringed- and a curved- medium voltage distribution network structure. The failure frequency on the medium-voltage distribution networks is higher than on the high-voltage networks. Figure 4 presents a failure of a mediumvoltage distribution board.

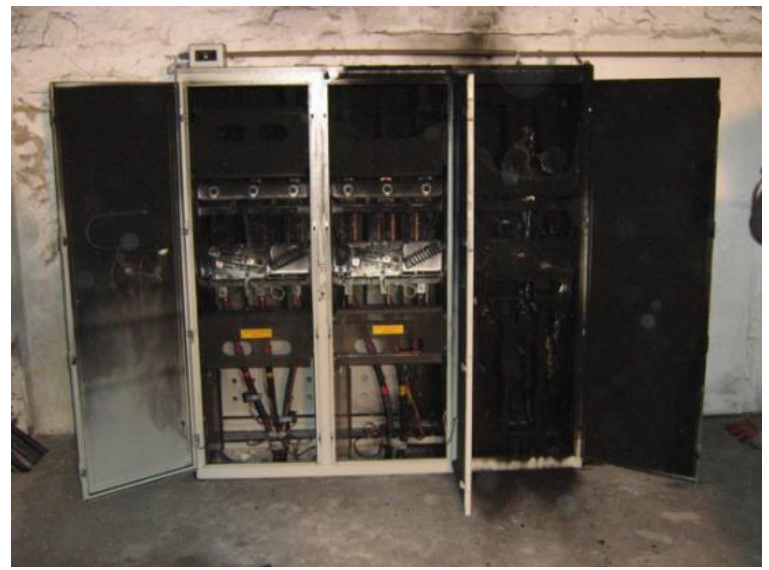

Figure 4

Identified MV failure location causing an outage at a consumer connection point [11] 


\subsection{Remote-controlled Switches and Short-Circuit Detectors}

The Hungarian power suppliers ELMÜ-ÉMÁSZ installed various remote signaling and remote controlled devices on the distribution networks in order to complete the delimitation of the failures and the speeding up of transfers, thus reducing the consumer disturbance and improving the SAIDI and SAIFI network quality index values.

Such a device is the remote controlled pole mounted disconnector (RPD) (Figure $5)$.

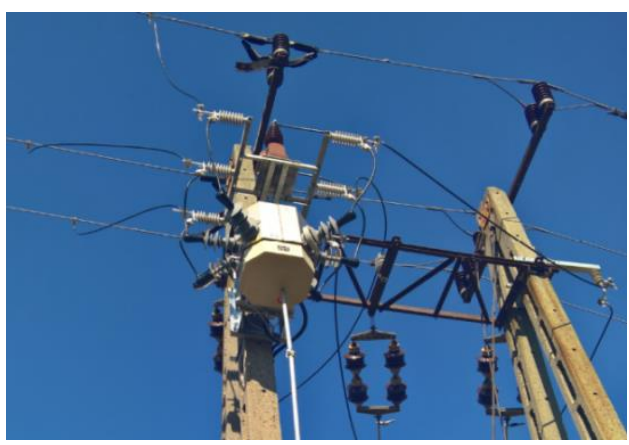

Figure 5

Remote controlled pole mounted disconnector on the ELMÜ-ÉMÁSZ network [11]

In addition to remote operation, the remote controlled pole mounted disconnector (Figure 5) provides information on the short-circuit currents and voltages flowing through it. Its application enables the automatic disengaging of shorted wires during the idle time of operation control through turning the pole mounted disconnector off.

Another device is the remote controlled switchgear on the distribution networks (RSD). This can ensure the possibility of remote operation by installing ex-post motors and current converters into the NERi, RM6 and similar devices (Figure 6).

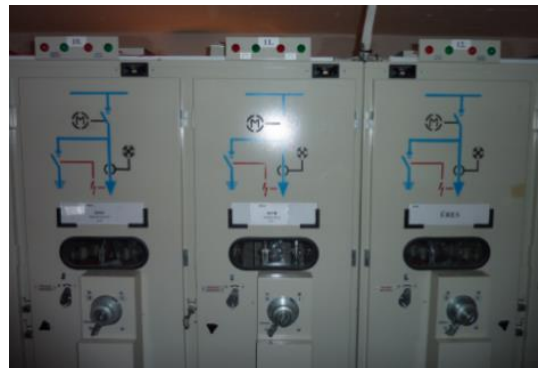

Figure 6

RSD-ized NERi type device on the ELMÜ-ÉMÁSZ network [11] 
Along with the remote signaling and operation devices, other devices with far lower investment needs are used today on medium voltage distribution networks enabling exclusively the identification of the failure location. The targeted positioning of these devices within the networks, e.g., at network junctions (Figure 7), can significantly facilitate and shorten the failure detection time consequently shortening the malfunction period.
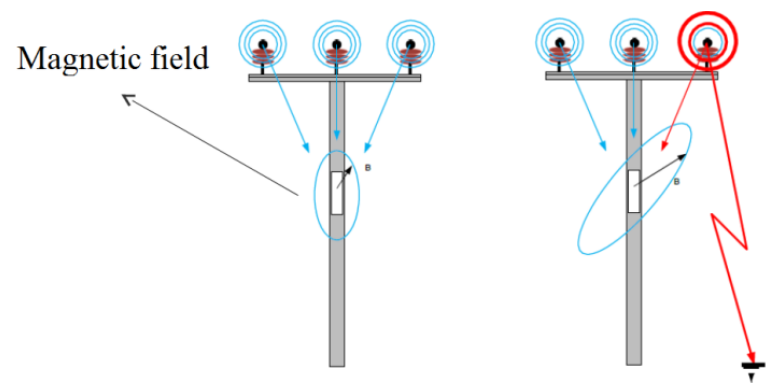

Figure 7

LineTroll R400D fault indicator (left: normal functioning, right: ground fault) [11]

According to the operational principle of the fault indicator (Figure 7), the fault perception of the device is based on the perception of the variation of the electromagnetic field below the line. This has to be installed $3 \mathrm{~m}$ below the middle line (Figure 7, encircled on the left-side figure).

Another solution for the same task is provided by the reinforced fault indicator pole (Figure 8):

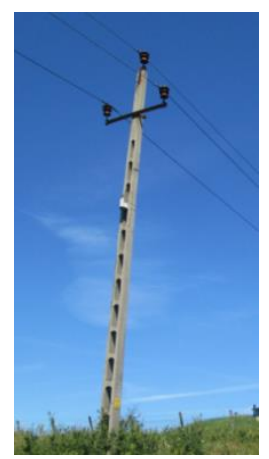

Figure 8

LineTroll R400D fault indicator installed on a reinforced concrete pole [11] 


\section{Developments Trends for Low-Voltage Distribution Networks}

In contrast to, the partially looped topology of the medium-voltage distribution networks, the radial or tree-like topological characteristics of the low-voltage distribution networks do not enable the use of such temporary solutions as in the case of the medium-voltage distribution networks. Due to the high anticipated costs, such a development is not to be expected for the future, since, according to its definition, "the radial network consists of main lines fed from the supply point and their laterals, whose lines are not in contact either with one another or with line fed from other supply points" $[8,9]$. Remote signaling and remote controlled devices are currently operating on low-voltage distribution networks only on a pilot basis. The significant number of SAIDI and SAIFI indicators stems precisely from the failures of low-voltage distribution networks, as given in Table 1.

Table 1

Unplanned consumer disturbance of ELMÜ-ÉMÁSZ Ltd. in 2017

\begin{tabular}{|c|c|c|c|c|c|c|c|}
\hline & $\begin{array}{c}\text { Number of } \\
\text { disturbances }\end{array}$ & $\begin{array}{c}\text { Duration } \\
\text { of outage } \\
\text { (hours) }\end{array}$ & $\begin{array}{c}\text { Number of } \\
\text { affected } \\
\text { consumers }\end{array}$ & $\begin{array}{c}\text { SAIDI } \\
\text { (mintes) }\end{array}$ & $\begin{array}{c}\text { SAIDI } \\
{[\%]}\end{array}$ & $\begin{array}{c}\text { SAIFI } \\
\text { (minutes) }\end{array}$ & $\begin{array}{c}\text { SAIFI } \\
{[\%]}\end{array}$ \\
\hline LV individual fault & 26500,0 & 65339,6 & 26500 & 0,0 & $1 \%$ & 4,0 & $2 \%$ \\
\hline LV medium fault & 9619,0 & 25512,7 & 336477 & 0,3 & $15 \%$ & 45,0 & $27 \%$ \\
\hline MV & 1783,0 & 5238,1 & 1965311 & 1,8 & $83 \%$ & 119,9 & $71 \%$ \\
\hline HV & 1,0 & 0,1 & 35896 & 0,02 & $1 \%$ & 0,1 & $0 \%$ \\
\hline Sum: & 37903,0 & 96090,4 & 2364184 & 2,2 & $100 \%$ & 169,0 & 1,0 \\
\hline
\end{tabular}

Due to the $15 \%$ SAIDI and the $27 \%$ SAIFI effect, the implementation of remote signaling devices on LV networks with lower investment costs is worth considering.

In the present paper we examine the automatic intervention possibilities as a further development of the LV distribution network operation. The basic idea stems from the application of this technology in Hungary since the 1980's for the fast and efficient handling of the temporary short-circuits in MV networks.

\subsection{The Reclose Function}

"When the reclose function is activated, the circuit breaker recloses after a previously specified time period following the defense action. If the defense continues to detect the short-circuit, the circuit breaker opens again. Following this and after another pre-set time period, the automatics will close the contacts of the circuit breaker again. The automatics seek to switch back two times (two cycles). The final release is activated if the short-circuit persists. Nowadays, reclose technology is already in use for MV networks. Its working principle and the pre-set time periods for MV networks are shown in Figure 9. 


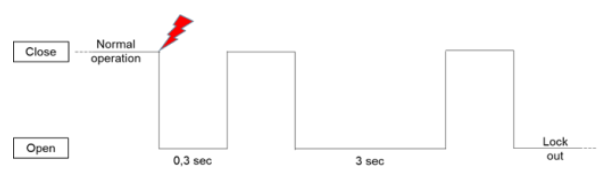

Figure 9

Working principle and pre-set time periods of the reclose function in case of MV networks

The implementation of the reclose function into the LV network will optimize the work of the electricians (they do not have to spend any more time going out to detect the faults in the network) and minimize the length of the LV power outages caused by short-circuits" [13].

\subsection{Reclose Function on the LV Network}

On LV networks, short-circuits are usually caused by external factors (e.g., tree branch touching the line, rain-related flashover, heavy wind, etc.) or by temporary overloads. Usually, the electrician sent to the location to resolve the outage only needs to change the fuse in phase 1,2 or 3, depending on the number of phases affected by the event. In this case, no further mechanical or electrical interventions are required. The development of the reclose function of the Smart Switchboard for LV distribution networks enables the reduction of temporary short-circuits to failures causing at most 3 minutes of consumer outage.

The steps of the intervention are shown on the flow chart in Figure 10.

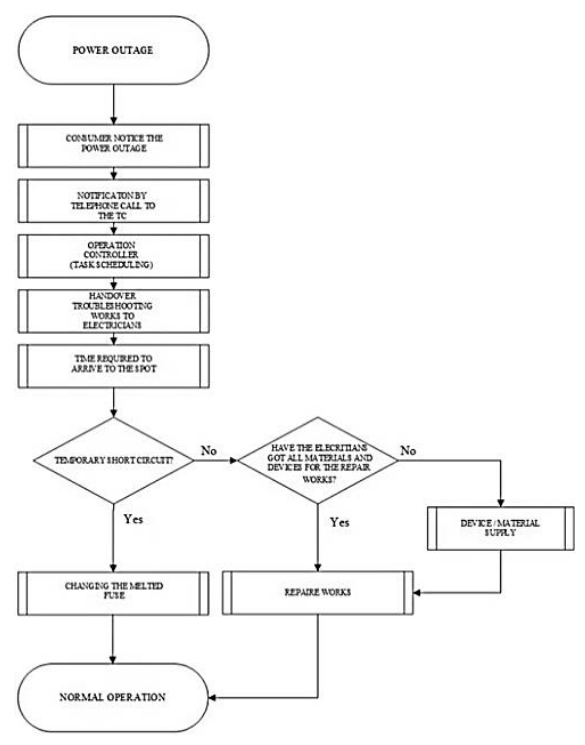

Figure 10

Flow chart of the current LV troubleshooting process [5] 
Figure 10 presents the current method of troubleshooting from the occurrence of the fault until the restoring of the normal operation.

\subsection{From the Smart Sensor to the SSB}

Currently, the fault localization on the LV distribution networks is done manually [5]. The lack of an automated practice for fault localization on the LV distribution network is due to the lack of a failure detecting device on the LV residential consumer network. Nevertheless, for the sake of automation, researchers and suppliers have developed new plans and pilot programs [9]. Intelligent consumption meters and low-voltage distribution boards equipped with smart sensors (Smart Switch Board - SSB) are opening new perspectives and enabling modern solutions for the localization of the LV network malfunctions.

\subsubsection{The Smart Sensors and the FLDa}

The data of the smart sensors [9] installed on the LV distribution networks - from intelligent consumption meters to smart functions built into the distribution board - created the possibility for the development of an algorithm for the fault localization. The algorithm is capable of localizing the eventual faults. It was introduced into the scientific discourse as the fault location determination algorithm (FLDa). Running the algorithm gives a one-line fault message containing the individual ID of the defective device, its address (coordinates), and the percentage of the determination accuracy for the identified failure. The results of the algorithm can be used as input to the current fault scheduling dispatcher system (i.e. the LV fault-sheet scheduling system - the LFS) [9].

\subsubsection{The Concept of the Smart Switchboard}

"The Smart Switchboard (SSB) concept stands for a remote controlled LV switchboard which uses a circuit breaker for the dismantling of the short-circuit current. The detection of the short-circuit current is carried out by using detection equipment together with a corresponding measurement analysis system. It is suitable for remote switch-on (circuit breaker activation) which, if necessary, can be turned to clogging mode. It contains the possibility of visible interruption point and earthing functions as well.

The visible interruption point and the earthing functions are required for ensuring the life, health and safety protection during maintenance, reconstruction, etc. works. The remote monitoring functions could actively or passively monitor the current, the voltage and the performance of the LV system. The implementation of an automatic recloser, a so-called reclose function into the SSB is also possible" [14]. 
We have used the 2014 and 2015 data of ELMÜ-ÉMÁSZ Ltd. for the efficiency analysis of the SSB system, i.e., for determining the actual SAIFI and SAIDI savings. The results were published in our previous articles [5].

"By carrying out the study of the efficiency, the research group determined the number of costumers for which it is worth upgrading the existing equipment. A cumulative efficiency function has been developed. This function shows the SAIDI improvement which could be achieved when changing some of the fuses to SSB's.

For example, in case $N=105$ customers:

$$
\text { Cumulative utility }=\sum_{N=105}^{N_{\max }}\left(N_{i} \cdot U_{i}\right)
$$

where $N$ is the number of customers in the LV network behind the fuse, $N_{\max }$ is the maximum number of customers behind a fuse, $N_{\mathrm{i}}$ is the number of customers affected by the power outage $i, U_{\mathrm{i}}$ is the duration of the power outage $i$ " [5].

The cumulative efficiency function is given in Figure 11.

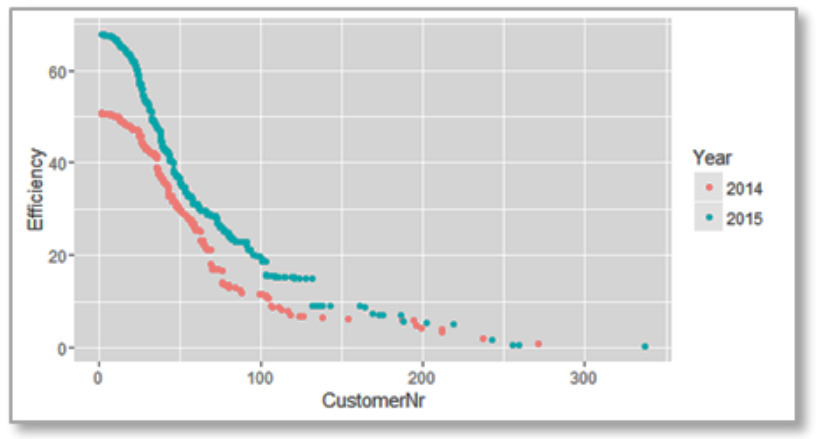

Figure 11

Efficiency in observations 2014/2015 [5]

The curve from Figure 11 regarding the measured data in year 2015 has been determined using regression analysis. To solve this problem the authors used the built-in polyfit function of the MATLAB software (fit type: poly34). The regression model was developed by using the 3 rd order polynomial regression according to which:

$C U(N)=74.526-0.8611 \cdot N+0.003317 \cdot N^{2}-0.000004095 \cdot N^{3}$

Hence, the present paper contains the theoretical approach for the previously published practical efficiency calculations. 


\subsection{The LV Fault-Sheet Scheduling System}

The decisions of the dispatchers operating the low-voltage distribution network and the fault address allocations are supported by IT systems. These software are based on the LV fault-sheet scheduling system (LFS). The LFS is not an IT software but a system describing the processes of receipt, processing and allocation of the faults.

Power suppliers take notice of the outages of the residential electricity supply network from the fault reports of the consumers. Phone calls are registered at the error reporting center (TeleCentrum - TC). These registered fault addresses and the related information is received by the dispatchers through the IT system. The dispatchers allocate the reparation of faults to the trouble shooting electrician teams on the basis of the received information and taking into account the professional experience of the team members. [9]

The implementation of smart sensors with FLDa and LFS can significantly accelerate the complete lead time of the LV fault management systems [9, 19]. This solution, in this specific form, deals exclusively with the processing of signals based on the remote signaling and does not take into account the possibility of remote and automatic intervention. The expected investment costs of these devices are elevated. Hence, their system integration can be taken into account only after a significant quality improvement of the networks [20, 21, 22].

\section{Implementation of the SSB in the LFS System}

The LFS system supplemented with the SSB system processes is shown in Fig. 12.

According to Figure 12, the process is started by $\tau$ and $\rho$ external excitations. The external excitation $\rho$ might be any external factor influencing the number and the professional composition of the teams of electricians involved in the repair of the breakdowns. It can also consist in the expiry of the shifts or in the beginning of new shifts and standby periods as well as in tasks from new work management responsibilities, in capacity requirements from higher plant management levels, in human factors (e.g., sickness, electrician's vehicle breakdown), etc.

Excitation $\tau$ might be any event resulting in LV malfunctions causing more than one consumer outage, e.g. network overload, external influences, fallen trees, flashover, etc.

For new $\tau$ excitations, the fault is currently still transmitted to the work management systems by the telecentre that is expected to be replaced by the smart sensors [9]. The task of the work management system is to compile the $1 \times \mathrm{m}$ sized $\overline{\bar{H}}$ matrix, extended with the $k$ parameters on the basis of the incoming information. 


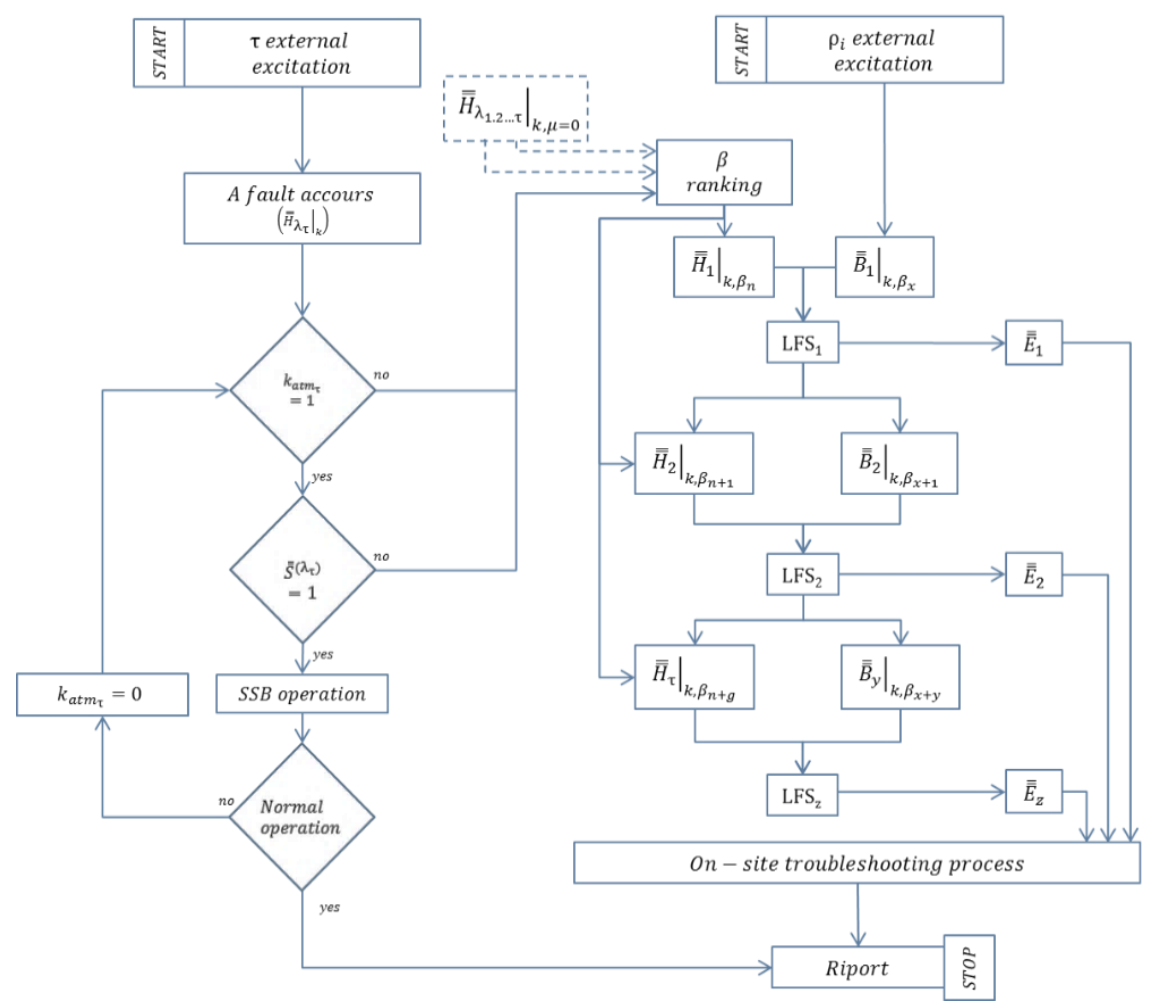

Figure 12

Implementation of the SSB in the LFS system

The $k$ parameter, of which $m$ are associated to the specific fault, might be the geographical location of the error (e.g., GPS coordinated), its priority or the number of affected consumers. The selection of these $k$ parameters for setting up the system was based on the requirement of the faulty addresses to be allocated to the electrician trouble shooting teams at the optimal location and in possession of all the necessary tools, professional exams, etc. E.g., the $\overline{\bar{H}}$ matrix transposition of the $\lambda^{\text {th }}$ incoming fault at $\tau$ location in the graph structure of the network (the transposition is for the purposes of transparent representation):

$\overline{\bar{H}}_{\lambda_{\tau}}^{T}=\left[\begin{array}{c}\text { Budapest } \\ 1037 \\ \text { Bécsi út } 96 / b \\ \text { KDÜL } \\ \text { Gyenes } \\ 42160 / 10 \\ \text { bus consumer } \\ 1 \text { fogyasztó } \\ \text { nem lakossági fogyasztó }(k) \\ \text { kábeles hálózat } \\ \text { prioritás:3 } \\ \text { 47.5338768 } \\ 19.0343905\end{array}\right]$ 
$\overline{\bar{H}}_{\lambda_{\tau}}^{T}$ is the illustration of the matrix. Its content structure is given by the users, in our case the power supplier's decision. The values of the line in the $\overline{\bar{H}}_{\lambda_{\tau}}^{T}$ matrix:

$\overline{\bar{H}}_{\lambda_{\tau}}^{T}=\left[\begin{array}{c}\text { city } \\ \text { postcode } \\ \text { address } \\ \text { substation } \\ \text { name of the MV line } \\ \text { transformer ID } \\ \text { LV circuit ID/ bus customers } \\ \text { number of the customers } \\ \text { type of the customers } \\ \text { network type } \\ \text { priority } \\ \text { north latitude GPS coordinate } \\ \text { east longitude GPS coordinate }\end{array}\right]$

If the specific fault affecting the location $\tau$ is of a transitional character (for further details, see Chapter 4.2), i.e.,

$k_{\text {atm }}=\left\{\begin{array}{l}1, \text { if } \overline{\bar{H}}_{\lambda_{\tau}} \text { is temporary faults } \\ 0, \text { if } \overline{\bar{H}}_{\lambda_{\tau}} \text { is not temporary faults }\end{array}\right.$

and there is an SSB at the specific $\tau$ fault location, i.e.,

$\overline{\bar{S}}\left(\lambda_{\tau}\right)=1$

where

$\overline{\bar{S}}^{\left(\lambda_{\tau}\right)}=\left\{\begin{array}{c}1, \text { if the node } \lambda_{\tau} \text { has } S S B \\ 0, \text { if the node } \lambda_{\tau} \text { nothas } S S B\end{array}\right.$

and

$k_{a t m_{\tau}} \cdot \overline{\bar{S}}^{\left(\lambda_{\tau}\right)}=\pi_{\tau}$

where $\pi_{\tau}$ represents a variable that can take only the value 0 or 1 .

In case of $\pi_{\tau}=1$, the SSB becomes operational and attempts to eliminate the fault. If it succeeds in doing so, then it sends a report to the plant management. If the fault remains in spite of the operation, then $k_{a t m_{\tau}}=0$, where $\pi_{\tau}=0$.

In case of $\pi_{\tau}=0,\left.\overline{\bar{H}}_{\lambda_{\tau}}\right|_{k}$ is incorporated into the $\beta$ ranking process. The process will introduce the fault address into a $\tau x k$ sized $\left.\overline{\bar{H}}_{\tau}\right|_{k, \beta_{n+g}}$ matrix on the basis of the evaluation of the $k$ parameters. [17] The $\beta$ values could stand for:

$\beta 1$ : Danger of death and risk of accidents

$\beta 2$ : High priority address (e.g., hospital)

$\beta 3$ : Malfunction affecting a high number of customers

$\beta 4$ : Malfunction affecting a low number of customers

$\beta 5$ : Malfunction affecting a single customer 
Hence, all faulty addresses received in the system that cannot be eliminated with the SSB, enters into a $\left.\overline{\bar{H}}_{\tau}\right|_{k, \beta_{n+g}}$ matrix.

Electricians currently on shift or on call form the matrix $\left.\overline{\bar{B}}_{y}\right|_{k, \beta_{x}}$. The faulty addresses of the system with the highest (numerically lowest) $\beta$ ranking, along with the $k$ parameters associated with the addresses, will be the first to enter into the faulty address scheduling algorithm $\left(\mathrm{LFS}_{1}\right)$. The result of running the $\mathrm{LFS}_{1}$ is entered into the $\overline{\bar{E}}_{1}$ matrix.

However, the $\overline{\bar{E}}_{1}$ results matrix contains not necessarily all the faults. It might happen that there will be $k$ parameter needs that can be fulfilled only if one of the electrician teams has completed the fault correction at a given address. E.g., it is possible that there are three fault addresses where climbing the pole is needed, but there is only one electrician with the required authorization to perform this task. These addresses, for which the $k$ parameter need cannot be immediately fulfilled, will be put into a waiting state (of course, these can also be de-scheduled on the basis of individual decisions).

In addition to the addresses forced into the waiting state the electricians who did not receive any address $\left(\left.\overline{\bar{B}}_{1}\right|_{k, \beta_{x+1}}\right)$ as well as the addresses with the lowest $\beta$ rankings $\left(\left.\overline{\bar{H}}_{\tau}\right|_{k, \beta_{n+1}}\right)$ will remain in the system. The $\mathrm{LFS}_{2}$ will complete its run with this input data and its result will be entered in the $\overline{\bar{E}}_{2}$ matrix.

This will go on for $z$ cycles. $z$ is reached when there is no more address left in the system. Then, the process will be put in standby mode until the next external excitation.

In the present case, the external excitation might be an electrician team becoming available or a new fault report. If a new external excitation occurs, the process restarts. That is to say, the $\mathrm{LFS}_{\mathrm{z}}$ completes its run in the case of any $\left.\overline{\bar{H}}_{\tau}\right|_{k, \beta_{n+g}}$ or $\left.\overline{\bar{B}}_{1}\right|_{k, \beta_{x}}$ change, thus also facilitating the shortening of the entire system runtime.

The final step of the fault correction administered by the SSB and completed by the electricians is the reporting to the plant management centers.

The flow chart in Figure 13 shows with green color the processes rendered superfluous in the fault elimination by the installation of the SSB and by the activation of its reclose function at the temporary fault location.

An additional advantage to the benefit of decoupling consists in the fact that, if the function is activated on the MV network with the parameters set on an empirical basis, then the complete run-time of the system will be less than 3 minutes; i.e. they will be considered as short-term disturbances and will not be taken into account in the SAIDI and SAIFI calculations (see Chapter 1). 


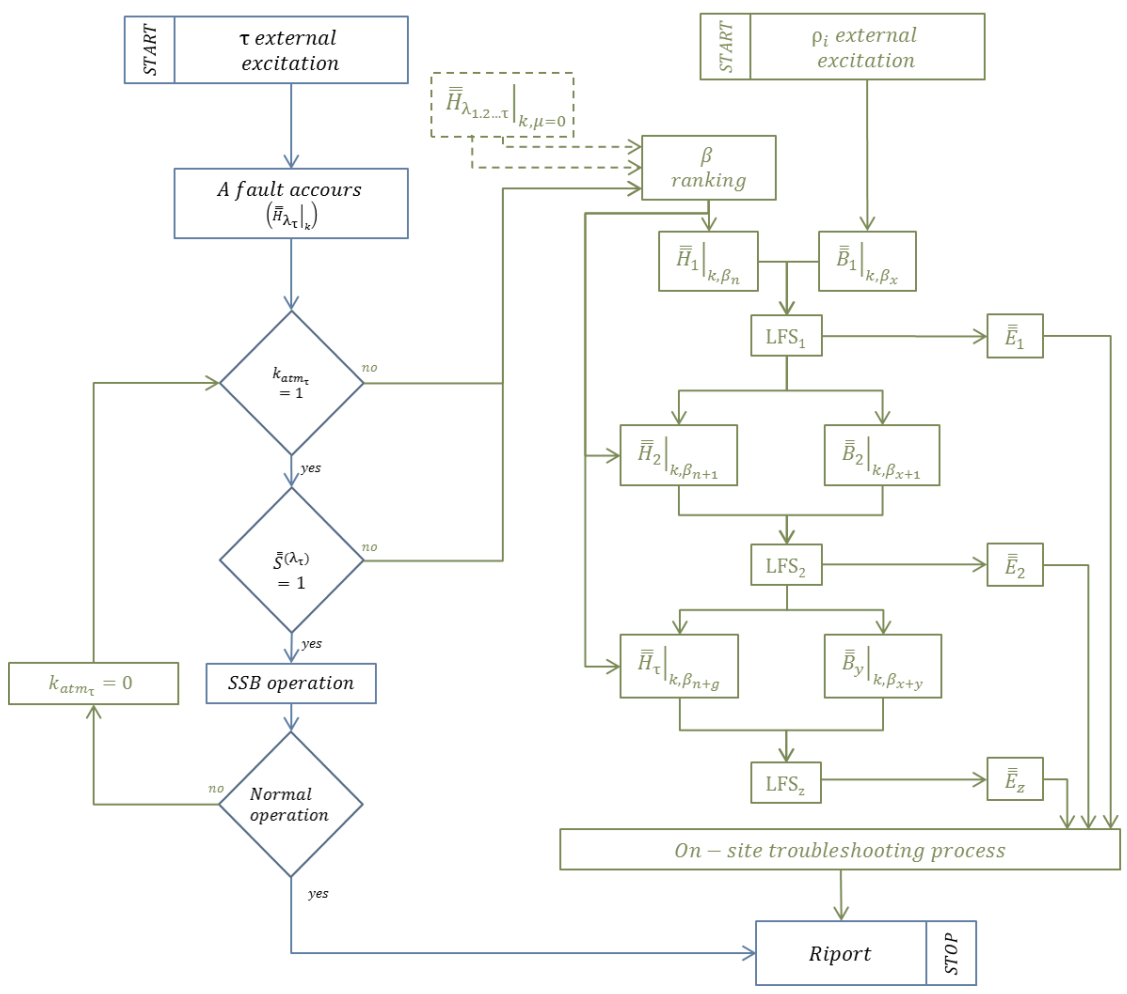

Figure 13

The effect of the SSB reclose function on the handling of temporary faults

\section{Conclusions}

This paper presented the smart switchboard devices and the theoretical advantages of their use in the LV distribution networks. This objective was derived from the existing technological developments and theoretical approaches. We have shown that the application of new results makes possible to be improve the network security on the network level through shortening the processes running time in case of faults due to temporary malfunctions.

\section{Acknowledgements}

The present paper was prepared at the Research Group of Applied Disciplines and Technologies in Energetics (AD\&TE) at the University Óbuda and it was supported by the ELMÜ Network Ltd. and ÉMÁSZ Network Ltd.

\section{三ł}

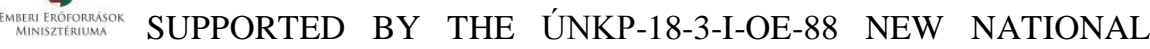
EXCELLENCE PROGRAM OF THE MINISTRY OF HUMAN CAPACITIES. 


\section{References}

[1] F. Faludi, L. Szabó: Operation and control of the electric power system, Budapest University of Technology and Economics, BMEVIVEM265, https://vet.bme.hu/sites/default/files/tamop/vivem265/out/html/ vivem265.html, 2012

[2] "1366-2012 - IEEE Guide for Electric Power Distribution Reliability Indices" Revision of IEEE Std. 1366-2003 (Revision of IEEE Std. 1366-1998), May 31 2012, DOI:10.1109/IEEESTD.2012.6209381

[3] K. Zou, W. W. Keerthipala, S. Perera: Saidi minimization of a remote distribution feeder, Australasian Universities Power Engineering Conference AUPEC, Perth, Australia, pp. 342-346, ISBN 9780646494883, 2007

[4] T Illés, G Lovics: Approximation of the Whole Pareto Optimal Set for the Vector Optimization Problem, Acta Polytechnica Hungarica, Vol. 15, No. 1, pp. $127-148,2018$

[5] J. Pálfi, M. Tompa, P. Holcsik: Analysis of the Efficiency of the Recloser Function of LV Smart Switchboards, Acta Polytechnica Hungarica, Vol. 14, No. 2, pp. 131-150, 2017

[6] G. Cordoş, M. Fülöp: Understanding audit reporting changes: introduction of Key Audit Matters, Accounting \& Management Information Systems/Contabilitatesi Informatica de Gestiune, Vol. 14, No. 1

[7] P. Yashodhan Agalgaonkar, J. Donald Hammerstrom: Evaluation of Smart Grid Technologies Employed for System Reliability Improvement: Pacific Northwest Smart Grid Demonstration Experience, Power and Energy Technology Systems Journal IEEE, Vol. 4, pp. 24-31, ISSN 2332-7707, 2017

[8] E.ON Hungária Zrt.: Distributor Policy on the Rules for Cooperation on Access to the Distribution Network, 3. modification, 4. 12, Budapest, 2009

[9] J. Pálfi: Applying Big Data Methods to Power Supply, PhD thesis, Doctoral School of Applied Informatics and Applied Mathematics, Óbuda Unievrsity, Budapest, 2018

[10] Gy. Morva: Electric Power Engineering, Education College, Book of TÁMOP4.1.2 A1 and TÁMOP-4.1.2 A2, 2012

[11] B. Czakó, G. Horváth: Operating systems and support systems, http://www.unimiskolc.hu/ elkborzo/uzemiranyitas_Czako_Horvath.pdf,2017

[12] A. Faludi, L. Szabó: Operation and control of the electric power system, Budapest University of Technology and Economics, Book of TAMOP 4.2.5

[13] P. O. Geszti: Electric Power Systems I., Textbook Publishing Company, Budapest, ISBN:963-17-6987-9, 1983

[14] J. Pálfi, P. Holcsik: New Database and Theoretical Model for Power Distribution Networks, Proceedings of the $9^{\text {th }}$ International Scientific Symposium on Electrical Power Engineering Elektroenergetika, pp. 539-544, 
Technical University of Košice Faculty of Electrical Engineering and Informatics, 2017

[15] J. Pálfi., P. Holcsik, L. Pokorádi: Determination of Customer Number by Matrix Operations in Case of Network Failure, IEEE $12^{\text {th }}$ International Symposium on Applied Computational Intelligence and Informatics (SACI 2018), Timisara, Romania, pp. 555-560, ISBN:978-1-5386-4639-7, 2018

[16] L. Pokorádi: Graph Theoretical Investigation of Network Structure System Scientific Bulletin Series C: Fascicle Mechanics, Tribology, Machine Manufacturing Technology, Vol. 2013, Issue 27, pp. 56-58, 2013

[17] M. Jocic, E. Pap., A. Szakál, D. Obradovic, Z. Konjovic: Managing Big Data Using Fuzzy Sets by Directed Graph Node Similarity, in Acta Polytechnica Hungarica, Vol. 14, No. 2, pp. 183-200, 2017

[18] T. Bagi: Measuring Current Distribution of Phase Conductor and Current Intesity Induced in Ground Wire on the $400 \mathrm{kV}$ Transmission Line, Proceedings of the $9^{\text {th }}$ International Scientific Symposium on Electrical Power Engineering Elektroenergetika, pp. 681-687, Technical University of Košice Faculty of Electrical Engineering and Informatics, 2017

[19] A. Dineva, A. R Várkonyi-Kóczy, V. Piuri, J. K. Tar, Point Cloud Processing with the Combination of Fuzzy Information Measure and Wavelets, Soft Computing Applications, Springer International Publishing, p. 584, 2018

[20] B. Niu, Y. Fan, H. Wang, L. Li, X. Wang, Novel bacterial foraging optimization with time-varying chemotaxis step, International Journal of Artificial Intelligence, Vol. 7, No. A11, pp. 257-273, 2011

[21] S.Vrkalovic, E. Lunca, I. Borlea, Model-free sliding mode and fuzzy controllers for reverse osmosis desalination plants, International Journal of Artificial Intelligence, Vol. 16, No. 2, pp. 208-222, 2018

[22] T. Haiddegger, L. Kovács, R-E. Precup, B. Benyó, Z. Benyó, S. Preitl, Simulation and control for telerobots in space medicine, Acta Astronautica, Vol. 181, No. 1, pp. 390-402, 2012 